\title{
Dynamic Analysis of Cantilever Structure for Catenary System
}

\author{
Li Shaopeng \\ Institute of Electrification, China Railway Design Corporation, Tianjin, China
}

\author{
Email address: \\ liseanh@163.com
}

\section{To cite this article:}

Li Shaopeng. Dynamic Analysis of Cantilever Structure for Catenary System. American Journal of Traffic and Transportation Engineering. Vol. 6, No. 3, 2021, pp. 52-57. doi: 10.11648/j.ajtte.20210603.11

Received: May 10, 2021; Accepted: May 24, 2021; Published: June 3, 2021

\begin{abstract}
Structural dynamics analysis is widely used in the fields of civil engineering, automobile manufacturing industry, Aerospace industry, and construction machinery. It is rarely applied in the railway transportation industry, especially the catenary arm structure. In this paper, a dynamic analysis of the cantilever structure is carried out, and the natural frequency of the cantilever structure is obtained. Through research, the frequency range that can cause the resonance of the cantilever structure is found and compared with the structural vibration frequency caused by the external load in the actual working condition. For this purpose, a finite element model of cantilever structure was created based on engineering practice. Static analysis was done by using finite element method to test the model's strength and stiffness. Then a dynamic model was made and modal analysis was done in order to get the first ten natural frequencies and corresponding modes. Resonance frequency was got by taking harmonic response analysis under the influence of external load. After all of these, a conclusion was made and the overall structure of the resonance phenomenon will not occur in actual situation which coincides with engineering actuality. It provides theoretical support for engineering practice. And also provides a theoretical basis for the follow-up structural anti-loose research.
\end{abstract}

Keywords: Finite Element Method, Nature Frequency, Static Analysis, Dynamic Analysis, Cantilever Structure

\section{Introduction}

Structural dynamics analysis is widely used in the fields of civil engineering, automobile manufacturing industry, Aerospace industry, and construction machinery. It can help people deepen their understanding of the characteristics of the structure itself. For example, it can let people know under what circumstances external forces will cause structural resonance, which in turn will affect the safety of the structure, and what measures can be taken to prevent such incidents from happening.

The resonance can cause loose parts, it would greatly reduce the reliability of the structure. More seriously, according to Xiao S, Luo Y's study, it can affect the current collection performance between the pantograph and the catenary [1]. It should be avoided as far as possible in the process of design and construction. When the pantograph passes through the positioning clamp, it would cause impact and vibration to the cantilever structure system. At the same time, the changes of wind load, contact line tension and other external loads would also have a certain impact on the whole structure system of the catenary. For the catenary system, under the normal driving condition, the vertical natural frequency " $\mathrm{f}$ " of the contact line is about $1 \mathrm{~Hz}$. At present, there are few researches on the analysis and calculation of the cantilever support structure and the resonance relationship between the cantilever support structure and the contact wire. Generally, they only rely on working experience, without theoretical support. There is no strict requirement for this in the national standard, which is often ignored and ignores the dynamic performance of the structure.

This article draws on Yang Y, El-Shabrawy M M, Hh A, DSA Júnior, Rodrigues $M$, et al.'s correction method of Timoshenko beam theory, and also refers to the way $\mathrm{Ttt} A$, Ssbca B, Do V D, Hiroaki K, Cruceanu I C et al's method of obtaining frequency response, analyzes and evaluates the dynamic performance of the overall structure system through modal analysis, harmonic response analysis and related mechanical calculations of the cantilever structure system, and verifies the degree of conformity between the model and the actual working conditions [1-3]. 


\section{Structural Static Model and Static Characteristics Analysis}

This paper selects the most unfavorable working condition, that is, the large-bounded cantilever structure system with anchor support as the analysis object. The distance between the base of the cantilever is $1.8 \mathrm{~m}$, the total length of the flat cantilever is $4.1 \mathrm{~m}$, and it is a steel cantilever structure. Establish a complete Solid Works entity model and simplify the model according to actual working conditions to complete the transformation of the finite element model. The cantilever and insulator of the finite element model are simulated by spatial beam elements, and the cantilever support is simulated by linkelements. Figure 1 is the SolidWorks solid model of the cantilever structure system, and Figure 2 is the simplified finite element model of ANSYS.

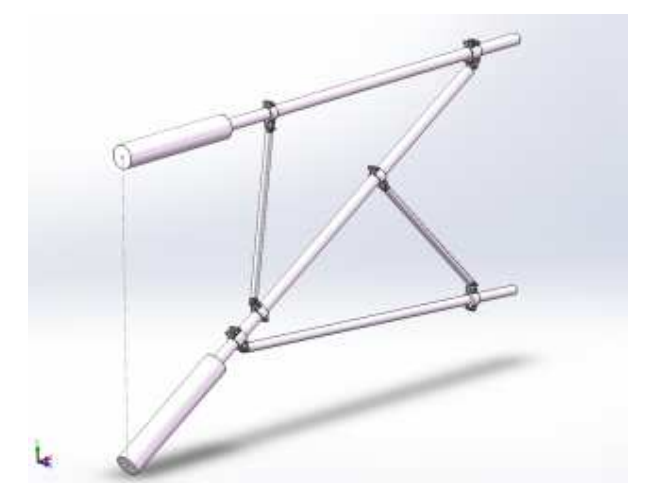

Figure 1. Solid modal for the cantilever structure system.

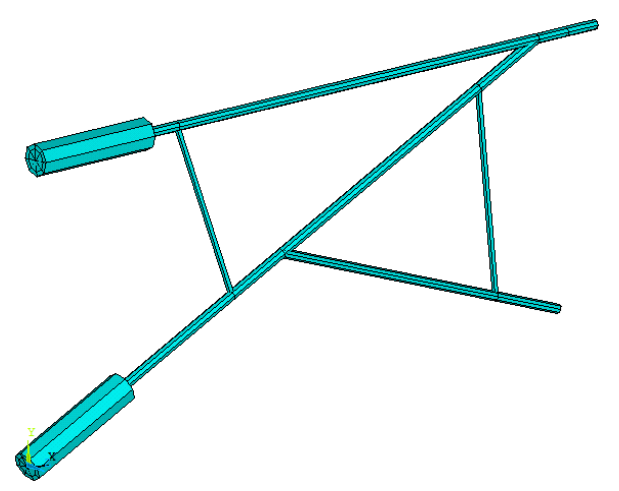

Figure 2. Finite element modal for the cantilever structure system

In the actual working state, considering the additional load caused by the tension of the contact line, weight, wind load, ice and snow load, and anchoring, the static analysis of the cantilever structure system is done.

Since this article aims to analyze the overall force of the wrist structure, in order to eliminate all local stresses that may be caused by modeling, the calculation results are more in line with the actual engineering, and the structural connections (bolts, positioning rings, etc.) are simplified as two-point coupling. Since the insulator structure is very easy to cause local stress during modeling, and its influence on the calculation results is minimal, it is simplified to a simple cylindrical structure, but its quality and size are consistent with the real situation.

The BEAM188 beam element based on Timoshenko beam theory [4-8] is used for simulation. The cantilever structure system model in this paper can be simplified as a continuous beam with spatial elastic support. According to related literature, the finite element equation of continuous beam element can be derived.

The nodal displacement function is:

$$
\{v\}=\left[1, x, x^{2}, x^{3}\right]\left[\begin{array}{cccc}
1 & 0 & 0 & 0 \\
0 & 1 & 0 & 0 \\
\frac{-3}{l^{2}} & \frac{-2}{l} & \frac{3}{l^{2}} & \frac{-1}{l} \\
\frac{2}{l^{3}} & \frac{1}{l^{2}} & \frac{-2}{l^{3}} & \frac{1}{l^{2}}
\end{array}\right]\left\{\begin{array}{c}
v_{i} \\
\theta_{i} \\
v_{j} \\
\theta_{j}
\end{array}\right\}=[N]\{\delta\}^{e}
$$

Where $v_{i}$ Displacement at element node $i$.

$\theta_{i} \longrightarrow$ Corner at element node $i$.

$v_{j} \longrightarrow$ Displacement at element node $j$.

$\theta_{j}-$ Corner at element node $j$.

$l$-Distance between nodes $i$ and $j$.

$[N]$ is the shape function matrix $N_{i 1}=1-\frac{3 x^{2}}{l^{2}}+\frac{2 x^{3}}{l^{3}}$, $N_{i 2}=x\left(1-\frac{2 x}{l}+\frac{x^{2}}{l^{2}}\right), N_{j 1}=\frac{3 x^{2}}{l^{2}}-\frac{2 x^{3}}{l^{3}}, N_{j 2}=x\left(\frac{x^{2}}{l^{2}}-\frac{x}{l}\right)$.

The relationship of element stress expressed by nodal displacement is:

$$
\left\{\sigma_{x}\right\}=E \varepsilon_{x}=E[B]\{\delta\}^{e}
$$

Where $E$ is the elastic modulus of the material.

The stiffness matrix [9] of the element expressed by the nodal displacement is:

$$
[K]^{e}=\iiint_{V}[B]^{T}[D][B] d x d y d z
$$

Considering that $[D]$ is a matrix related to the elastic modulus of the material, and the cross-sectional moment of inertia is $I=\iint y^{2} d y d z$. The relationship between the nodal force and the nodal displacement acting on the element is established by the principle of virtual work, that is, the element stiffness equation is:

$$
\{F\}^{e}=[K]^{e}\{\delta\}^{e}
$$

Where $\{F\}^{e}$ is the element node force component array, from which $[K]^{e}$ can be solved.

In summary, the equation when calculating with the finite element method can be described as:

$$
[K]\{\delta\}=\{F\}
$$

Where $[K]$ is the overall stiffness matrix of the structure, which is composed of the stiffness matrix of each element; 
$\{\delta\}$ is the nodal displacement array; $\{F\}$ is the nodal load array. By bringing the actual displacement boundary conditions into equation (5), the node displacement of the element can be solved, and then the required stress and strain can be obtained through the relational expression established by the element characteristic analysis.

This paper uses ANSYS finite element calculation software to analyze and calculate the strength and static stiffness of the model. The results are shown in Figure 3:

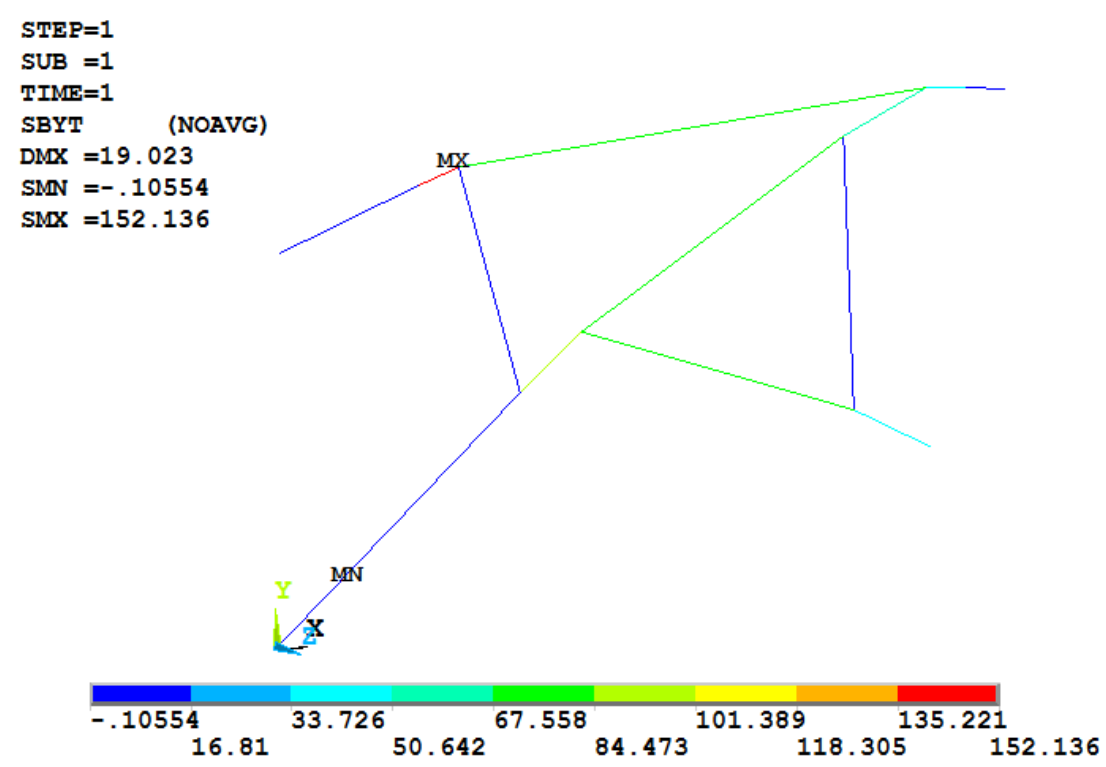

Figure 3. Maximum stress for the cantilever structure system.

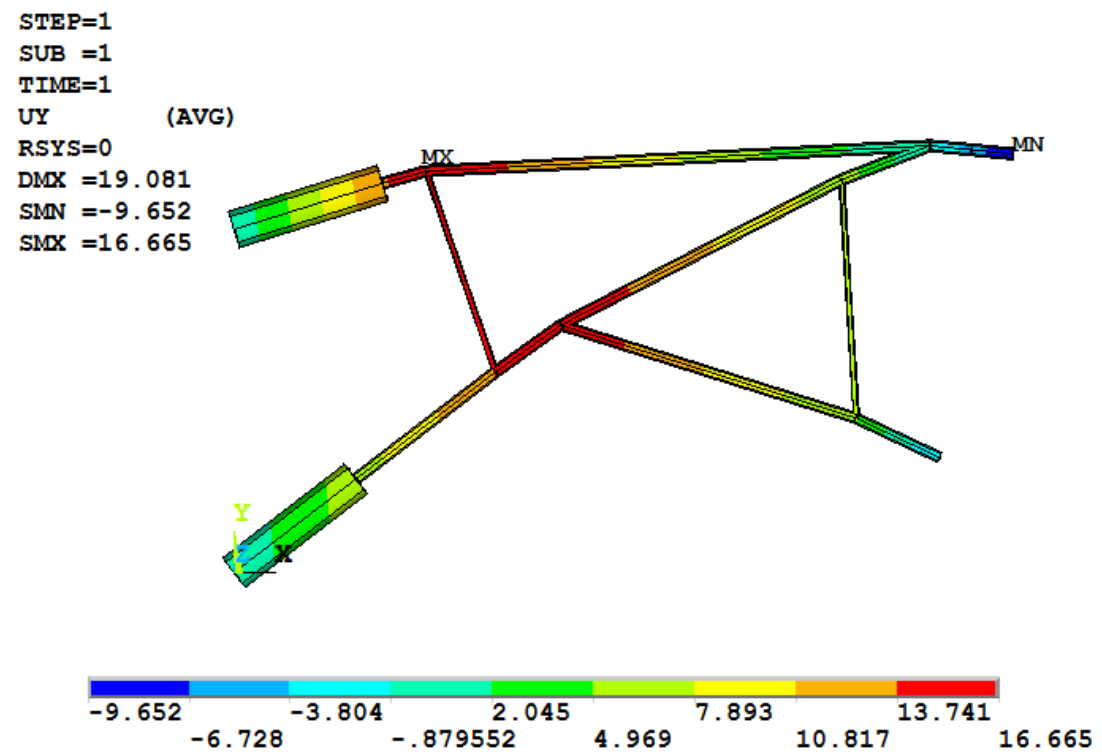

Figure 4. Static stiffness for the cantilever structure system.

It can be seen from the above figure that the maximum stress of the cantilever structure system appears at the connection between the flat cantilever and the cantilever support, and its value is 152.13MP. Due to the steel arm structure, the allowable stress of its material is $245 \mathrm{MPa}$, which meets the design requirements. The maximum deflection occurs at the connection between the flat cantilever and the support, and its value is $16.665 \mathrm{~mm}$. The design specification requires that the deflection should not be greater than $0.7 \%$ of the length of the wrist arm, which also meets the design requirements.

\section{Structural Dynamic Model and Dynamic Characteristic Analysis}

The dynamic finite element equation of the structural system is $[10,11]$ :

$$
M \ddot{x}+C \dot{x}+K x=F
$$

In the formula, $M, C, K$ are the mass, damping and stiffness matrix of the structural system respectively, $x 、\{\dot{x}\} 、\{\ddot{x}\} 、\{F\}$ 
are the displacement, velocity, acceleration and nodal load array of the structural system respectively. By solving this equation, the inherent characteristic parameters and dynamic response parameters of the vibration system can be obtained.

In this paper, the finite element model of the cantilever structure system is taken as an example, and its modal analysis and harmonious response analysis are carried out by finite element method.

When performing modal analysis, the structure is regarded as a free vibration system that is not affected by external forces, and since damping has little effect on the natural frequency $[12,13]$ and mode shape of the structure, the natural frequency and mode shape can be solved according to the undamped free vibration. The vibration equation is:

$$
[M]\left\{x^{\prime \prime}\right\}+[K]\{x\}=\{0\}
$$

If the free vibration of an elastic body is decomposed into the superposition of a series of simple harmonic vibrations, the solution of equation (7) can be set as:

$$
\{x\}=\{A\} \sin (p t+\phi)
$$

In the formula, $p$ is the angular frequency, $\phi$ is the initial phase angle, and $\{A\}$ is the non-zero amplitude array. By substituting formula (8) into formula (7), the formula for the natural frequency and mode shape of the structure can be solved:

$$
\left([K]-p^{2}[M]\right)\{A\}=\{0\}
$$

The modal analysis [14] is done on this model, and get the first 10 natural frequencies of the system as shown in Table 1. The main vibration mode corresponding to the natural frequencies of the $1 \mathrm{st}, 4 \mathrm{th}, 5 \mathrm{th}, 7 \mathrm{th}$, and 10 th order is the horizontal vibration of the structure along the traveling direction of the train. The main vibration mode corresponding to the second natural frequency reflects the torsional vibration of the structure along the direction of the flat arm. The $3 \mathrm{rd}, 6 \mathrm{th}$, 8 th, and 9th order main vibration modes reflect the vertical vibration of the structure on the vertical plane. Since the cantilever structure can be regarded as a simple truss system, its first 10 natural frequencies and vibration modes mainly depend on the torsional and bending stiffness of the cantilever. The modal analysis results are shown in Table 1:

Table 1. The first ten natural frequencies of cantilever.

\begin{tabular}{ll}
\hline Order/n & Frequency/Hz \\
\hline 1 & $5.47 \mathrm{E}-6$ \\
2 & 6.739 \\
3 & 8.3672 \\
4 & 9.8963 \\
5 & 13.524 \\
6 & 32.114 \\
7 & 37.988 \\
8 & 54.184 \\
9 & 91.279 \\
10 & 101.99 \\
\hline
\end{tabular}

Use ANSYS software to analyze the harmonic response of the structure $[15,16]$, and find out the vibration frequency that has the greatest influence on the dynamic performance of the structure under the action of external loads (especially the moment when the pantograph passes, wind load and contact wire tension changes). Under the action of dynamic loads with different excitation frequencies, the maximum vertical displacement point of the cantilever system and the maximum peak value of the vertical displacement response of the anchor positioning clip appear in the third mode, as shown in Figure 6. Therefore, the third-order mode is most likely to cause resonance of the structure. For the natural frequency" f" of the vertical direction of the contact line under normal working conditions, the value is about $1 \mathrm{~Hz}$. The natural frequency of the wrist structure in this case in the vertical direction is $8.3672 \mathrm{~Hz}$. It can be seen that it will not cause resonance of the overall wrist-arm structure system and meet the design requirements.

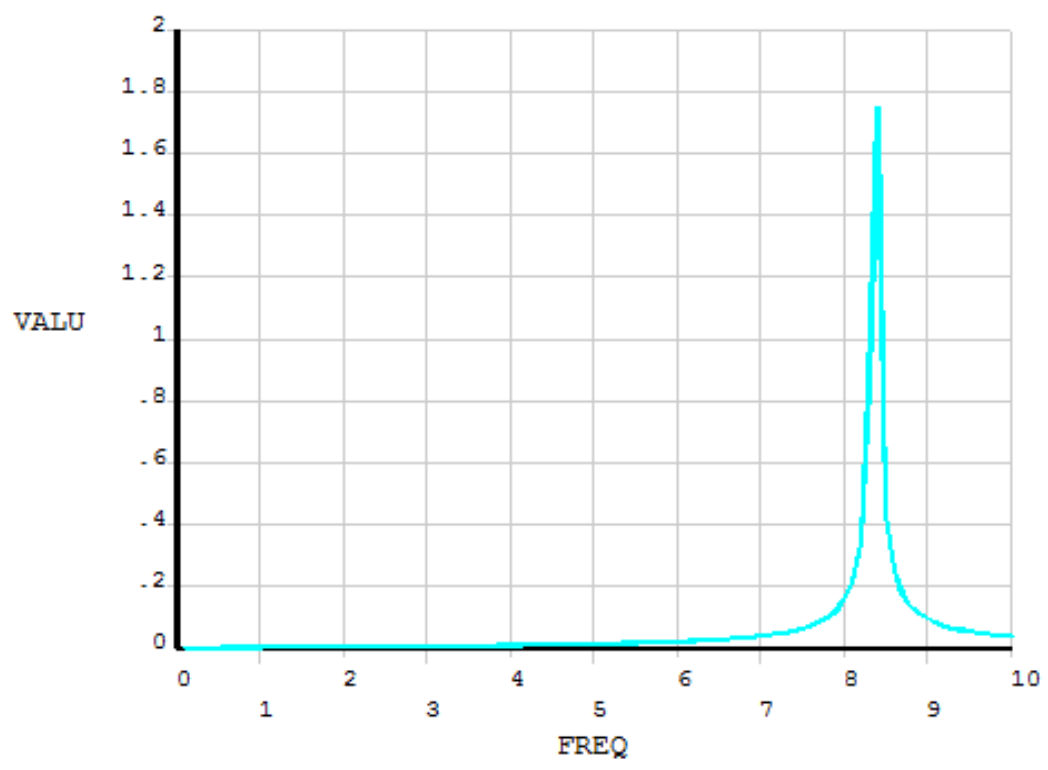

Figure 5. Vertical displacement response of maximum vertical displacement point. 


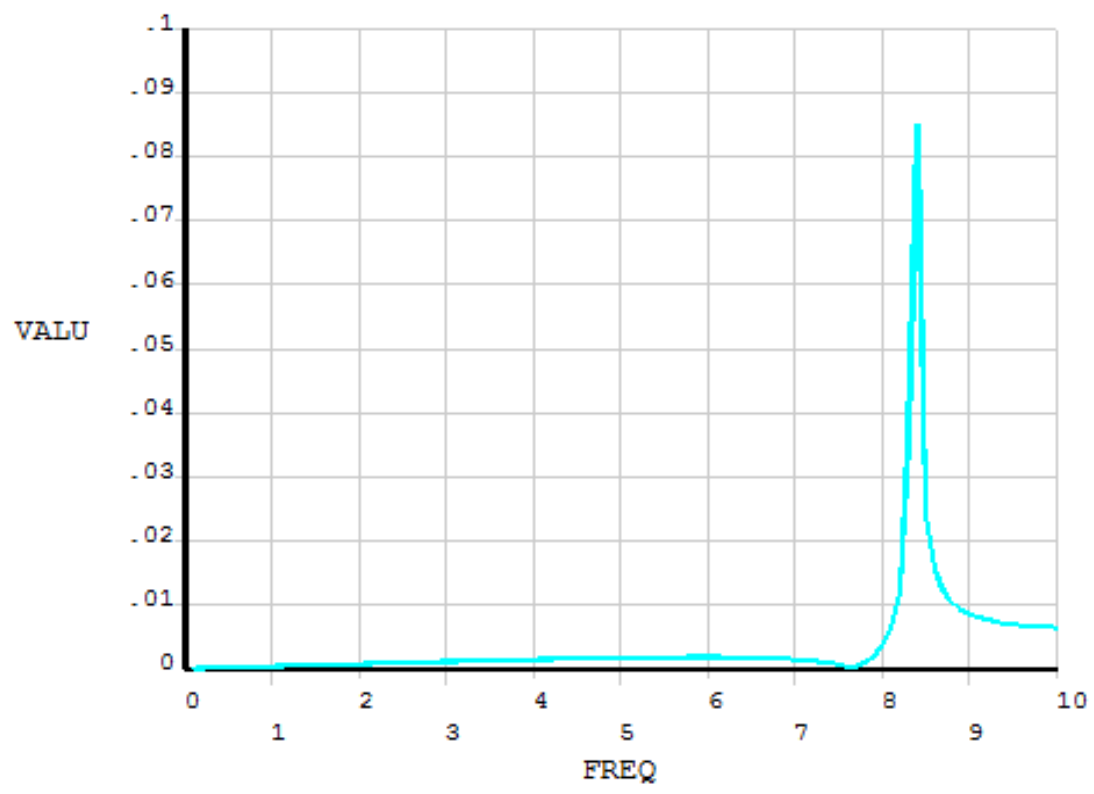

Figure 6. Vertical displacement response of holder for tube with out-of-running C. W clamp.

\section{Conclusion}

In this paper, the solid model and finite element model of the cantilever structure system are established according to the actual engineering. Through the analysis and calculation of the model, the static strength and static rigidity of the structural system were checked, and the dynamic analysis of the structural system under the external load was carried out. The natural frequency of the structure is determined through modal analysis and harmonic response analysis. From the above calculations, the static strength and static stiffness of the model established in this paper meet the requirements of use, and the resonance phenomenon that affects the overall reliability will not occur under the action of external loads (contact wire tension, pantograph, wind load, etc.). The actual situation is consistent, providing theoretical support for the actual engineering project. At the same time, through the analysis and calculation of this paper, the basic form of structural vibration can also be obtained, which has certain guiding significance for the study of anti-loosening of bolts and nuts.

\section{References}

[1] Xiao S, Luo $\mathrm{Y}$, $\mathrm{Wu} \mathrm{J}$, et al. An Investigation into the Characteristics of a Novel Rotatable Pantograph Catenary System for High-Speed Trains [J]. Journal of Electrical Engineering and Technology, 2021, 16 (7): 1-10.

[2] Li M, Ma M, Cao Z, et al. Dynamic response analysis of train-induced vibration impact on the Probhutaratna pagoda in Beijing $[\mathrm{J}]$. Earthquake Engineering and Engineering Vibration, 2021, 20 (1): 223-243.

[3] AntunesP, J Ambrósio, Pombo J, et al. A new methodology to study the pantograph-catenary dynamics in curved railway tracks [J]. Vehicle System Dynamics, 2019: 1-28.
[4] Yang Y, Wu Q. WITHDRAWN: Analysis of dynamic fracture mechanics parameters of one-point bend specimens based on modified Timoshenko's beam theory [J]. International Journal of Pressure Vessels \& Piping, 2016: S0308016116300539.

[5] El-Shabrawy M M, Abdeen M, Nassar M, et al. Analysis of Tapered Timoshenko and Euler-Bernoulli Beams on an Elastic Foundation with Moving Loads [J]. Journal of Mathematics, 2021.

[6] HhA, Tya B, Le V, et al. Dynamic and static isogeometric analysis for laminated Timoshenko curved microbeams [J]. Engineering Analysis with Boundary Elements, 2021, 128: 90-104.

[7] Terzi V G. Soil-structure-interaction effects on the flexural vibrations of a cantilever beam [J]. Applied Mathematical Modelling, 2021.

[8] DSA Júnior, Ramos A, Freitas M M. Energy decay for damped Shear beam model and new facts related to the classical Timoshenko system [J]. Applied Mathematics Letters, 2021, 120(41/245): 107324.

[9] Rodrigues M, Burgos R B, Martha L F. A Unified Approach to the Timoshenko 3D Beam-Column Element Tangent Stiffness Matrix Considering Higher-Order Terms in the Strain Tensor and Large Rotations [J]. International Journal of Solids and Structures, 2021.

[10] TrcfA, Atb B. Dynamic analysis of failure paths of truss structures: Benchmark examples including material degradation [J]. Mechanical Systems and Signal Processing, 158 .

[11] C S X Z A B, D C W B, C Z Z S A B. Engineering analysis of the dynamic characteristics of an electrical jacket platform of an offshore wind farm under seismic loads [J]. Applied Ocean Research, 112.

[12] TttA, Qhpb C, Ntb C. Static and free vibration analyses of functionally graded porous variable-thickness plates using an edge-based smoothed finite element method [J]. Defence Technology, 2020, 17(6). 
[13] Ssbca B, Srp B, Bka C. Modal testing and evaluation of cracks on cantilever beam using mode shape curvatures and natural frequencies [J]. Structures, 2021, 32: 1386-1397.

[14] Do V D, Le T P, Beakou A. Operational modal analysis of mechanical systems using transmissibility functions in the presence of harmonics [J]. Journal of Science and Technology in Civil Engineering (STCE) - NUCE, 2019, 13 (3): 1-14.

[15] Hiroaki K, Hayasaka K, Watanabe M. Frequency response of a cantilevered rectangular sheet under harmonic forced excitation in three-dimensional uniform flow [J]. Journal of Fluids and Structures, 103
[16] Cruceanu I C, Sorohan T. Determination of the Harmonic Response of a Railway Wheelset using the Finite Element Analysis Method[J]. Procedia Manufacturing, 2020, 46:173-179.

\section{Biography}

Li Shaopeng, Born in 1988, Male, Engineer of China Railway Design Corporation. Graduated in June 2014, Southwest Jiaotong University, Mechanical Design and Theory, Master of Engineering, 